\title{
Impacto del dragado de sedimentos del puerto de Salaverry en el ecosistema marino litoral
}

\section{Impact of dredging of sediments of the Salaverry Port on the litoral marine ecosystems}

\author{
Carlos A. Bocanegra García \\ Universidad Nacional de Trujillo, Tujillo, Perú \\ E-mail: carlosbocanegra2013@gmail.com
}

Recibido el 14 de noviembre del 2016; aceptado el 10 de diciembre del 2016

DOI: https://doi.org/10.33017/RevECIPeru2016.0009/

\section{Resumen}

Se evaluó el impacto del dragado de sedimentos en el puerto de Salaverry, principal centro de transporte de productos agrícolas, mineros, petróleo entre otros. El último dragado realizado en agosto del 2014, consistió en remover 1 163,000 m3 de sedimentos del fondo marino, con una draga hidráulica que succionó y depositó el material dragado en el entorno cercano del área portuaria, a la izquierda como derecha de la plataforma. Se determinó los principales contaminantes químicos $(\mathrm{Pb}, \mathrm{Zn}, \mathrm{Cd}, \mathrm{Cu})$ indicativos de la actividad portuaria. El estudio se realizó en ambas zonas de depósito del material dragado, de donde se tomó muestras de sedimentos y agua superficial, aves y peces capturados. En ambas zonas los valores de los metales pesados superaron el límite máximo permitido de acuerdo a los stándares internacionales, lo cual significa efectos negativos sobre el ecosistema marino costero y coloca en riesgo la salud pública.

Descriptores: Impacto ambiental, contaminación portuaria, metales pesados, ecosistema litoral.

\section{Abstract}

The impact of dredging of sediments in the port of Salaverry, main center of transport of agriculture products, minerals, petroleum and others was evaluated. The last dredging carried out in august of 2014, removed 1 $163,000 \mathrm{~m} 3$ of the marine bottom sediments, with a hidraulic dredge which, were sucked out and lay aside wear the port área, at the left the right of fishermen platform. Main chemical contaminants were determined $(\mathrm{Pb}, \mathrm{Zn}, \mathrm{Cd}, \mathrm{Cu})$ indicatives of the port activity . Investigations was carried out in both zones, where samples of the sediments and superficial wáter, birds and fishes were taken. The values of heavy metals were higher than the maximum permited limit according the international standars, performing negative effects on the coastal marine ecosystems making a risk to the public healt.

Keywords: Impact, chemical, marine ecosystems

\section{Introducción}

La Región La Libertad, cuenta con tres puertos, el de Pacasmayo, Malabrigo y Salaverry. El puerto de Salaverry se encuentra ubicado a $12 \mathrm{~km}$ de la ciudad de Trujillo y que por su fácil acceso permite el desarrollo de actividades como el embarque de productos agrícolas (principalmente del proyecto chavimochic), harina de pescado, fertilizantes, azúcar, arroz y concentrados de minerales, así como la pesca industrial y artesanal. Actividades que 
generan residuos propios del transporte que terminan impactando negativamente el ecosistema

marino costero, principalmente el fondo donde se depositan. Sin embargo uno de los problemas del puerto Salaverry es su constante sedimentación, vale decir la acumulación de sedimentos que impiden su operatividad, por lo que tiene que ser dragado.

Se tiene documentado a partir del año 1966 a 1999, un total de 19 millones de $\mathrm{m} 3$ de sedimento dragados en la dársena del puerto, con un promedio anual de $360000 \mathrm{~m}^{3} / a n ̃ o$. El puerto es dragado regularmente por la draga Marinero Rivas con más de 35 años de operación y tecnológicamente obsoleta según el Consorcio Haskoning - Indesmar [1]., y en el 2014, se dragó $1163000 \mathrm{~m}^{3}$, según el Diario La República, D.S. No. 019-2014-PCM [2], haciendo un total aproximado de 20 millones 163 mil metros cúbicos de sedimento removido del fondo.

Existen diversas investigaciones que mencionan que las actividades de dragado necesarias para poder mantener un calado adecuado de los canales de navegación en los puertos generan importantes impactos ambientales en los ecosistemas costeros, al provocar, modificaciones de los patrones locales de circulación, transporte de material suspendido y cambios en las condiciones ambientales del agua y contaminación como sostiene Gonzales et al [3].

Uno de los impactos más significativos está referido a la los contaminantes químicos que se introducen a los sistemas acuáticos asociándose al material en suspensión que al sedimentarse se incorporan en el fondo, donde permanecen hasta ser removidos; no obstante que los contaminantes asociados a los sedimentos se consideran generalmente no biodisponibles, su remoción durante el dragado tiene efectos que potencialmente incrementan su biodisponibilidad y son un riesgo para la vida de la biota según Winger et al [4]. Pero, además, el riesgo se amplía a la salud pública, pues los habitantes consumen los recursos marinos como peces, mariscos, algas; del mismo utilizan las playas para descanso, el agua para bañarse, situación que hasta ahora no ha sido abordada, como menciona Gonzales et al [3].

El ecosistema marino litoral de Salaverry, donde se ubica el puerto, no escapa a esta realidad, pues desde el inicio de sus operaciones se presentaron problemas de sedimentación del canal, hecho que generó que, a partir en la década del 70 , se realicen no solo dragados sino la construcción y ampliación sistemática de un super espigón o molón contenedor de arena de 500 metros iniciales a 1050 metros, alternativa que no resolvió el problema, tal como lo menciona Bocanegra $[5,6,7]$.

Estudios sobre la calidad ambiental del ecosistema marino litoral en Salaverry, están mayormente referidos a condiciones de contaminación por aguas residuales, tal como el de Perez [8], Imarpe [9, 10], Romero [11], Gómez [12].

Considerando los antecedentes del dragado a la que es sometida el puerto y tomando en cuenta que, en agosto del 2014, se realizó un dragado de 1 millón 300 mil metros cúbicos de sedimentos, el mismo que no contó con el respectivo estudio de impacto ambiental que exige la normatividad, se planteó evaluar el impacto de este dragado en el ecosistema referido a los sedimentos y agua superficial, a peces y aves que conforman dicho ecosistema, asumiendo como hipótesis que la remoción de los sedimentos con más de 40 años depositados en el fondo marino, definitivamente, contaminarían a los componentes del ecosistema en mención.

\section{Material y Métodos}

El área de estudio (Fig. 1) comprendió la zona litoral de la playa de Salaverry de influencia directa con el puerto donde atracan los busques y donde se desarrollan los dragados. Se estableció dos puntos de muestreo, uno a la izquierda de la plataforma o muelle (zona B) y otra a la derecha (zona A), pues en ambas zonas son arrojadas los sedimentos dragados.

El material de estudio lo constituyó muestras de agua y sedimento superficial del litoral, peces y aves capturadas en las zonas de estudio en horas de marea baja. Las muestras de sedimentos se tomó $200 \mathrm{~g}$ con una espátula a una profundidad de $10 \mathrm{~cm}$, las mismas que se depositaron en bolsas plásticas, previo al análisis de metales pesados, fueron secados. Las muestras de agua superficial se tomaron con botellas de $200 \mathrm{ml}$, para lo cual se ingresó al mar a unos $20 \mathrm{~m}$. Para el caso de aves y peces, después de su captura, se procedió a someter las muestras a la técnica de espectrometría de absorción atómica [13]. 


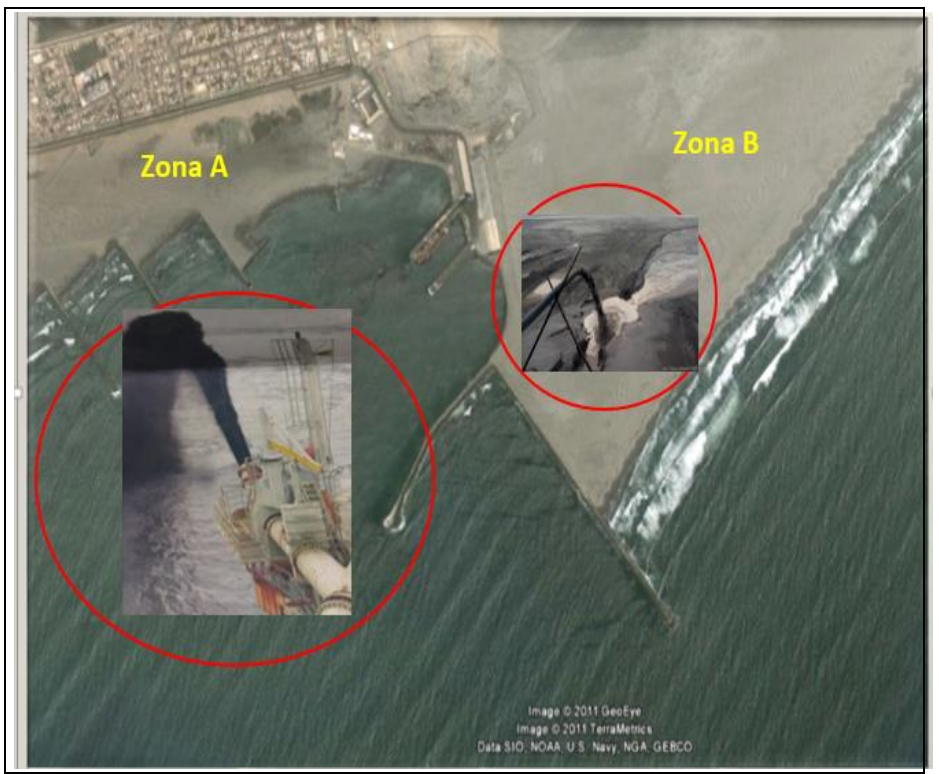

Figura 1: Área de estudio y localización de las zonas de muestreo.

\section{Resultados y discusión}

En la Tab. 1, se muestran las concentraciones de los metales pesados encontrados en las muestras de sedimento y agua superficial, músculo de aves y peces, para la zona B (izquierda del muelle). Los valores obtenidos en toda la zona de estudio revelan que $\mathrm{Pb}, \mathrm{Cu}$ y $\mathrm{Zn}$, fueron los metales con mayor concentración en las muestras obtenidas.

Tabla 1: Concentración de metales pesados ( $\mathrm{Cu}, \mathrm{Pb}$, $\mathrm{Zn}, \mathrm{Cd}$ ), en ecosistema al sur de puerto Salaverry, abril - mayo 2015.

\begin{tabular}{|c|c|c|c|c|}
\hline \multicolumn{2}{|l|}{ parámetro } & \multirow{2}{*}{$\begin{array}{c}\begin{array}{c}\text { muestra1 } \\
\text { abril }\end{array} \\
0.186\end{array}$} & \multirow{2}{*}{$\begin{array}{c}\begin{array}{c}\text { muestra } 2 \\
\text { mayo }\end{array} \\
0.050\end{array}$} & \multirow{2}{*}{$\begin{array}{c}\text { limites máximo } \\
\text { internacionales } \\
0.0500\end{array}$} \\
\hline agua & $\mathrm{Cu}$ & & & \\
\hline superficial & $\mathrm{Pb}$ & 0.246 & 0.324 & 0.0081 \\
\hline & $\mathrm{Zn}$ & 0.015 & 0.030 & 0.0810 \\
\hline & $\mathrm{Cd}$ & & 0.098 & \\
\hline \multirow{4}{*}{$\begin{array}{c}\text { sedimento } \\
\text { arena superficial }\end{array}$} & $\mathrm{Cu}$ & 8.100 & 10.940 & 108.0 \\
\hline & $\mathrm{Pb}$ & 4.920 & 18.660 & 30.2 \\
\hline & $\mathrm{Zn}$ & 32.320 & 66.000 & 124.0 \\
\hline & $\mathrm{Cd}$ & & 0.880 & \\
\hline \multirow{4}{*}{$\begin{array}{c}\text { musculo de pez } \\
\text { bagre } \\
\text { qaleichthvs peruvianus }\end{array}$} & $\mathrm{Cu}$ & 0.093 & 6.160 & 30.000 \\
\hline & $\mathrm{Pb}$ & 0.5171 & 0.070 & 2.000 \\
\hline & $\mathrm{Zn}$ & 0.1231 & 16.130 & 40.000 \\
\hline & $\mathrm{Cd}$ & & 0.220 & \\
\hline \multirow{4}{*}{$\begin{array}{l}\text { musculo de ave } \\
\text { piquero } \\
\text { sula variegata }\end{array}$} & $\mathrm{Cu}$ & & 18.000 & \\
\hline & $\mathrm{Pb}$ & & 1.010 & \\
\hline & $\mathrm{Zn}$ & & 30.100 & \\
\hline & $\mathrm{Cd}$ & & 0.280 & \\
\hline \multirow{4}{*}{$\begin{array}{l}\text { musculo de pez } \\
\text { caballito de mar }\end{array}$} & $\mathrm{Cu}$ & & 9.40 & 30.000 \\
\hline & $\mathrm{Pb}$ & & 2.75 & \\
\hline & $\mathrm{Zn}$ & & 52.70 & \\
\hline & $\mathrm{Cd}$ & & 0.74 & \\
\hline
\end{tabular}

Tabla 2: Concentración de metales pesados ( $\mathrm{Cu}, \mathrm{Pb}$, $\mathrm{Zn}, \mathrm{Cd}$ ) en el ecosistema al norte del Puerto de Salaverry, Abril, Mayo 2015

\begin{tabular}{|c|c|c|c|c|}
\hline \multicolumn{2}{|l|}{ parámetro } & \multirow{2}{*}{$\begin{array}{c}\begin{array}{c}\text { muestra1 } \\
\text { abril }\end{array} \\
0.186 \\
\end{array}$} & \multirow{2}{*}{$\begin{array}{c}\begin{array}{c}\text { muestra } 2 \\
\text { mayo }\end{array} \\
0.050 \\
\end{array}$} & \multirow{2}{*}{$\begin{array}{c}\text { limites máximo } \\
\text { internacionales } \\
0.0500\end{array}$} \\
\hline agua & $\mathrm{cu}$ & & & \\
\hline superficial & $\mathrm{pb}$ & 0.246 & 0.324 & 0.0081 \\
\hline & $\mathrm{zn}$ & 0.015 & 0.030 & 0.0810 \\
\hline & $\mathrm{cd}$ & & 0.098 & \\
\hline \multirow{4}{*}{$\begin{array}{c}\text { sedimento } \\
\text { arena superficial }\end{array}$} & $\mathrm{cu}$ & 8.100 & 10.940 & 108.0 \\
\hline & $\mathrm{pb}$ & 4.920 & 18.660 & 30.2 \\
\hline & $\mathrm{zn}$ & 32.320 & 66.000 & 124.0 \\
\hline & $\mathrm{cd}$ & & 0.880 & \\
\hline \multirow{4}{*}{$\begin{array}{c}\text { musculo de pez } \\
\text { bagre }\end{array}$} & $\mathrm{Cu}$ & 0.093 & 6.160 & 30.000 \\
\hline & $\mathrm{pb}$ & 0.5171 & 0.070 & 2.000 \\
\hline & $\mathrm{zn}$ & 0.1231 & 16.130 & 40.000 \\
\hline & cd & & 0.220 & \\
\hline \multirow{4}{*}{$\begin{array}{c}\text { musculo de ave } \\
\text { piquero } \\
\text { sula variegata }\end{array}$} & $\mathrm{Cu}$ & & 18.000 & \\
\hline & $\mathrm{pb}$ & & 1.010 & \\
\hline & $\mathrm{zn}$ & & 30.100 & \\
\hline & $c d$ & & 0.280 & \\
\hline \multirow{4}{*}{$\begin{array}{l}\text { musculo de pez } \\
\text { caballito de mar }\end{array}$} & $\mathrm{cu}$ & & 9.40 & 30.000 \\
\hline & $\mathrm{pb}$ & & 2.75 & 2.000 \\
\hline & $\mathrm{zn}$ & & 52.70 & 40.000 \\
\hline & $\mathrm{cd}$ & & 0.74 & \\
\hline
\end{tabular}

En la Tab. 2 se muestran las concentraciones de los metales pesados encontrados en las muestras de sedimento y agua superficial, músculo de aves y peces, para la zona B (izquierda del muelle). Los valores obtenidos en toda la zona de estudio revelan que $\mathrm{Pb}, \mathrm{Cu}$ y $\mathrm{Zn}$, fueron los metales con mayor concentración en las muestras obtenidas.

Se conoce que el dragado es la remoción, succión transporte y descarga de material del fondo marino utilizando para ello dragas. El principio general de las dragas hidráulicas es mezclar los sedimentos con agua para formar un líquido el cual es succionado, bombeado y transportado a través de las tuberías y finalmente depositado en un área preestablecida. En general es aceptado que todos los métodos de dragado y descarga de material dragado tienen el potencial para generar directa o indirectamente ciertos impactos negativos al ambiente. Esos impactos generan cambios en las características físicas, químicas y biológicas de los ecosistemas. Los potenciales impactos negativos que generan son: impactos sobre la calidad del agua, suspensión y distribución de sedimentos contaminados, impactos sobre peces, flora y otros organismos y cambios físicos del fondo acuático, según Landaeta [3].

$\mathrm{Si}$ analizamos los resultados obtenidos en el presente trabajo, coincidimos completamente, pues todos los componentes del ecosistema marino litoral de Salaverry se encuentra contaminado por el dragado. La presencia de metales pesados en el 
agua superficial, se atribuye que tanto durante como después del dragado y la descarga, los sedimentos son removidos del fondo, los cuales quedan suspendidos en la superficie o columna de agua.

En este caso particular los sedimentos, además de ser removidos, han sido transportados y depositados en zonas con acceso directo a la población humana. $\mathrm{Si}$ bien es cierto que los sedimentos gruesos se sedimentan rápidamente, los finos, como arena fina, limo y arcillas, permanecen en suspensión que pueden cubrir grandes áreas al ser transportados por las corrientes y las olas. Algunos estudios han reportado que los polvos o nube de sedimentos del dragado se dispersaron hasta un radio de 5 kilómetros cuadrados, generando turbidez y, por ende, reducción de la penetración de la luz necesaria para los procesos de fotosíntesis y cambios en el calor de radiación, según Morton, en Landaeta [14].

En el agua superficial, se encontró alteración en sus características químicas, por la presencia de trazas de metales pesados. Se conoce que el agua es el mayor vehículo de transporte de contaminantes y el medio en el cual esos contaminantes pueden desarrollar reacciones químicas y físicas, hecho que explica la presencia de estos metales en los sedimentos. En consecuencia, tal como lo afirma Landaeta [3], los procesos de dragado y descarga no incorporan nuevos contaminantes al medio acuático: simplemente tienen el potencial para poner en suspensión y distribuir los sedimentos contaminados, tal como ha quedado demostrado en esta investigación.

En los peces y aves marinas capturadas en las zonas de estudio, también se encontró presencia de metales pesados, atribuible a que durante el dragado y descarga se generan alteración de las características del agua y sedimento, sobre todo a nivel de nutrientes; por lo cual se recomienda hacer una caracterización biológica previa de los sedimentos a dragar, hecho que no se realizó. En el caso particular del pez "chita", ésta es carnívora y vive en la columna de agua, a diferencia del pez "bagre", "borracho y "tramboyo", que son bentónicos y tiene contacto directo con los sedimentos del fondo.

Estos resultados han confirmado lo que en su momento se dijo: de no tomarse en cuenta lo mencionado y la exigencia del estudio de impacto ambiental requerido de acuerdo a Ley, definitiva- mente, se produciría contaminación al ecosistema marino litoral, según Bocanegra [7], puesto que el material a dragar está permanentemente expuesto a los impactos negativos. Ya se tiene conocimiento de que los dragados y descarga del material removido deben realizarse bajo ciertas condiciones como confinar la zona a dragar, evitar la suspensión de los sedimentos, depositar los sedimentos dragados en áreas confinadas en tierra y luego incinerarlas, hecho que no se realizó y allí las consecuencias.

Al parecer no se ha tomado en cuenta que el mar peruano se caracteriza por los afloramientos costeros, los que son responsables de la riqueza de recursos hidrobiológicos, son los afloramientos que dan inicio a la cadena trófica del mar, por cuanto ponen a disposición los nutrientes de aguas profundas, que ascienden. Dijimos que el dragado de sedimentos y posterior deposición en el fondo marino o en el litoral, al ser removidos, se dispersará en el mar. El dragado y posterior deposición en el fondo marino, ha generado problemas serios para el ecosistema marino litoral y los recursos hidrobiológicos allí existentes. Este problema no es exclusividad nuestra, ya ha sido tratado en otras partes del mundo. Esto ya ocurrió en el dragado del rio Sena, Rio Támesis, incremento del puerto de Santos, Recuperación de la bahía de Chesapeak, y así otros cien lugares más alrededor del mundo. Actualmente este trabajo es realizado retirando el dragado hacia tierra [3, 15].

\section{Conclusiones}

El dragado de $1163000 \mathrm{~m}^{3}$ de sedimentos en el puerto de Salaverry (Trujillo), principal centro de transporte de productos agrícolas, mineros, petróleo, realizado en agosto del 2014, generó impactos negativos en el ecosistema marino litoral, que se evidenció en contaminación química con metales pesados en los componentes del agua y sedimentos superficial, peces y aves del entorno, tanto en el lado norte como el del lado sur del área portuaria.

Los contaminantes químicos ( $\mathrm{Pb}, \mathrm{Zn}, \mathrm{Cd}, \mathrm{Cu}$ ) superaron el límite máximo permitido de acuerdo a los estándares internacionales, hecho que significa riesgo para la salud pública.

\section{Referencias}

[1] Consorcio haskoning - indesmar, 2008 Informe de sedimentación

[2] Diario La República, 2014, D.S. No. 0192014 
[3] M. Gonzales, M. Rodríguez, D. López y A. Vázquez. 2006 Evaluación de la contaminación en sedimentos del área portuaria y zona costera de Salina Cruz, Oaxaca, México. Interciencia, versión impresa ISSN 0378-1844

[4] PV. Winger, PJ. Lasier, DH. White, JT. Seginak 2000 Effects of contaminants in dredge material from the lower Savannah river. Arch. Env. Contam. Toxicol. 38: 128136

[5] C. Bocanegra. 2011 Destrucción del litoral de Trujillo: Impacto de la transformación de la costa en la geomorfología litoral. Sociedad Geográfica de Lima.

[6] C. Bocanegra 2013 Crónica de un daño Ambiental Impune: Erosión costera de Trujillo. Editorial universitaria de la Universidad Nacional de Trujillo.

[7] C. Bocanegra. 2014 El Retorno del Desierto: Trujillo al 2050. Editorial universitaria de la Universidad Nacional de Trujillo

[8] J. Pérez Estrella. 2016 Contaminación en la playa de Salaverry_por aguas residuales se las pozas de oxidación, Distrito de Salaverry, La Libertad. Tesis para optar el Título de Biólogo Pesquero. Universidad Nacional de Trujillo, Perú.

[9] IMARPE, 2010 Estándares de calidad ambiental en la región La Libertad. Callao, Perú.

[10] IMARPE, 2011 Estándares de calidad ambiental en la región La Libertad. Callao,
Perú.

[11] C.B. Romero, 2016 Concentración de Coliformes totales y Escherichia coli en agua de mar de la playa de Salaverry. Tesis para optar el Título de Biólogo Pesquero. Universidad Nacional de Trujillo.

[12] A. D. Gómez, 2016. Calidad Ambiental de la zona litoral de puerto Salaverry, Libertad. Tesis para optar el Título de Biólogo Pesquero. Universidad Nacional de Trujillo.

[13] E. Perkin. 1976 Analytical Methods for Atomic Absortion Spectrophotometry. Perkin Elmer. EEUU. 122 pp.

[14] Landaeta, http://docplayer.es/14364080Potenciales-impactos-ambientalesgenerados-por-el-dragado-y-la-descarga-delmaterial-dragado-resumen.html

[15] C. Molina, C. Ibañez , François-Marie Gibon 2012 Proceso de biomagnificación de metales pesados en un lago hiperhalino (Poopó,Oruro, Bolivia):Posible riesgo en la saluddeconsumidoreshttp://www.scielo.org.b o/scielo.php?script=sci_arttext\&pid=S1605. 